Supporting Information

\title{
Effect of the Ni and NiO interface layer on the energy
}

\section{performance of core/shell $\mathrm{CuO} / \mathrm{Al}$ systems}

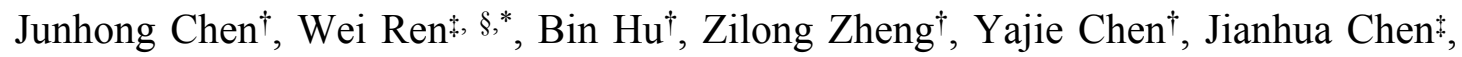
Chunpei $\mathrm{Yu}^{\dagger}$, Changkun Song ${ }^{\dagger}$, Jiaxin Wang ${ }^{\dagger}$, Wenchao Zhang ${ }^{\dagger, *}$

† School of Chemical Engineering, Nanjing University of Science and Technology, Nanjing 210094, P.R. China

$\$$ Science and Technology on Applied Physical Chemistry Laboratory, Shaanxi Applied Physics and Chemistry Research Institute, Xi'an 710061, China

$\S$ State Key Laboratory for Manufacturing Systems Engineering, Xi'an Jiaotong University, Xi'an 710049, China

${ }^{*}$ Correspondence authors: Wenchao Zhang, Wei Ren

Email: zhangwenchao@njust.edu.cn, rw0192@163.com

Tel: $+862584315515 \quad$ Fax: +862584315857

Number of pages: 7

Number of figures: 7 


\section{Content}

1. Materials and methods

2. XRD patterns of $\mathrm{CuO} @ \mathrm{NiO}$ nanowires film at different chemical bath deposition times (Figure S1)

3. EDS spectra for the three nanowires thermite film systems (Figure S2)

4. EDS line scan spectra of $\mathrm{CuO} @ \mathrm{NiO} / \mathrm{Al}$ (Figure $\mathrm{S} 3$ )

5. DSC curves of the three nanowires thermite film systems at various heating rates and corresponding linear fit result (Figure S4-S6)

6. $\mathrm{DSC}$ curves of $\mathrm{CuO} / \mathrm{Al}$ at different $\mathrm{Al}$ deposition time (Figure $\mathrm{S} 7$ ) 


\section{EXPERIMENTAL SECTION}

Materials. The chemical reagents $\mathrm{KOH}(\geq 90.0 \%), \mathrm{NiSO}_{4} \cdot 6 \mathrm{H}_{2} \mathrm{O}(\geq 98.0 \%), \mathrm{K}_{2} \mathrm{~S}_{2} \mathrm{O}_{4}$ ( $\geq 99.5 \%$ ), acetylacetone ( $\geq 99.0 \%)$, anhydrous ethanol ( $\geq 99.7 \%), \mathrm{H}_{2} \mathrm{SO}_{4}(95.0-98.0 \%)$ and ammonium hydroxide (24\%-25\%) were purchased from Sinopharm Chemical Reagent Co., Ltd. A platinum (Pt) electrode ( $\geq 99.9 \%)$ was obtained from Xuzhou Zhenghao Electronic Technology Co., Ltd. Al and Ni sputtering targets with a purity of $99.999 \%$ were supplied by Zhongnuo New Material Co., Ltd. Cu foils were used as substrates from Sinopharm Chemical Reagent Co., Ltd.

Preparation of $\mathrm{CuO} / \mathrm{Al}$ nanowire thermite films. Anodization was carried out in a double-electrode system with the pretreated $\mathrm{Cu}$ foil as the working electrode and a $\mathrm{Pt}$ electrode $\left(2 \times 2 \mathrm{~cm}^{2}\right)$ as the counter electrode. The distance is set to $2 \mathrm{~cm}$ between the $\mathrm{Cu}$ anode and $\mathrm{Pt}$ cathode. Then, the pretreated $\mathrm{Cu}$ foil, half of which was immersed into a $3 \mathrm{M} \mathrm{KOH}$ solution with a $2 \times 2 \mathrm{~cm}^{2}$ reaction area, was electrochemically anodized at a constant current density of $3 \mathrm{~mA} \cdot \mathrm{cm}^{-2}$ at room temperature. After anodizing for 20 minutes, the resultant blue $\mathrm{Cu}(\mathrm{OH})_{2}$ films were heated at $200^{\circ} \mathrm{C}$ for $3 \mathrm{~h}$ to obtain a dark brown layer of $\mathrm{CuO}$ nanowires on the surface of the $\mathrm{Cu}$ foils. Finally, $\mathrm{Al}$ was deposited onto the $\mathrm{CuO}$ skeleton by magnetron sputtering to obtain the $\mathrm{CuO} / \mathrm{Al}$ nanowire thermite film. The Al deposition time was set as $15 \mathrm{~min}, 30 \mathrm{~min}$ and $45 \mathrm{~min}$ to control the thickness. After thermal analysis (Figure S7), the optimized Al deposition time was determined to be $30 \mathrm{~min}$. All magnetron sputtering processes were performed under argon conditions, as the vacuum level of the working chamber was maintained at $8 \times 10^{-4} \mathrm{~Pa}$.

Preparation of the $\mathrm{CuO} @ \mathrm{Ni} / \mathrm{Al}$ nanowire thermite films. The preparation protocol for the $\mathrm{CuO} @ \mathrm{Ni} / \mathrm{Al}$ nanowire thermite film is similar to that of the $\mathrm{CuO} / \mathrm{Al}$ nanowire thermite film. It should be noted here that $\mathrm{Ni}$ is deposited onto the $\mathrm{CuO}$ skeleton for 1 min before the sputtering of Al. The $\mathrm{CuO} @ \mathrm{Ni} / \mathrm{Al}$ nanowire thermite film is finally prepared with $\mathrm{Ni}$ as an interface layer. 
Preparation of the $\mathrm{CuO} @ \mathrm{NiO} / \mathrm{Al}$ nanowire thermite films. The $\mathrm{Cu}(\mathrm{OH})_{2}$ films were immersed into a $100 \mathrm{~mL}$ mixed solution of $1 \mathrm{M} \mathrm{NiSO}_{4} \cdot 6 \mathrm{H}_{2} \mathrm{O}$ and $0.25 \mathrm{M} \mathrm{K}_{2} \mathrm{~S}_{2} \mathrm{O}_{4}$ with another $10 \mathrm{~mL}$ of ammonia under constant stirring for $10 \mathrm{~min}$. The reaction proceeded at room temperature to form the precursor nanostructures, which were subsequently washed with deionized water and dried at $300^{\circ} \mathrm{C}$ for $2 \mathrm{~h}$ in an oven before forming the core/shell $\mathrm{CuO} / \mathrm{NiO}$ nanowire film. Finally, $\mathrm{Al}$ was deposited onto the $\mathrm{CuO} / \mathrm{NiO}$ skeleton in a manner similar to the $\mathrm{CuO} @ \mathrm{Ni} / \mathrm{Al}$ thermite film preparation to obtain the $\mathrm{CuO} @ \mathrm{NiO} / \mathrm{Al}$ nanowire thermite film.

Characterization. XRD (Bruker, D8 Advanced) with monochromatized $\mathrm{Cu} \mathrm{K \alpha}$ radiation $(\lambda=0.15406 \mathrm{~nm})$ was used to investigate the crystal structures of all the samples. The surface morphology, elemental analysis and composition of the samples were probed by using field emission SEM (FESEM, Hitachi, S-4800), EDS (Oxford Instruments) and TEM (JEOL, JEM-2100), respectively. Additionally, the EDS was calibrated with pure metal ( $\mathrm{Al}, \mathrm{Cu}$ and $\mathrm{Ni}$ ) before the test. The heat release values were examined by DSC (Mettler Toledo) with a temperature range from $100{ }^{\circ} \mathrm{C}$ to $1000{ }^{\circ} \mathrm{C}$ at a heating rate of $10^{\circ} \mathrm{C} \cdot \mathrm{min}^{-1}$ with an argon flow rate of $30 \mathrm{~mL} \cdot \mathrm{min}^{-1}$. The combustion pressure was measured in a closed reactor with a constant volume of $12.56 \mathrm{~mm}^{3}$. For a typical measurement, $5 \mathrm{mg}$ of powder of each nanothermite was placed inside the closed reactor with an embedded bridge wire at the bottom, to which a constant current of $10 \mathrm{~A}$ was applied for ignition. The dynamic pressure from combustion was recorded through a pressure transducer on the top of the reactor, which was connected to an oscilloscope. The ignition performance was studied by a pulse laser (Beamtech, DAWA-350). The wavelength, pulse width and incident laser energy were set at 1064 $\mathrm{nm}, 100 \mathrm{~ns}$ and $167 \mathrm{~mJ}$, respectively. When the laser pulse acted on the sample, the whole ignition progress was recorded by a high-speed camera (Redlake Motion Xtra, HG-100K) that runs at 50000 frames per second. 


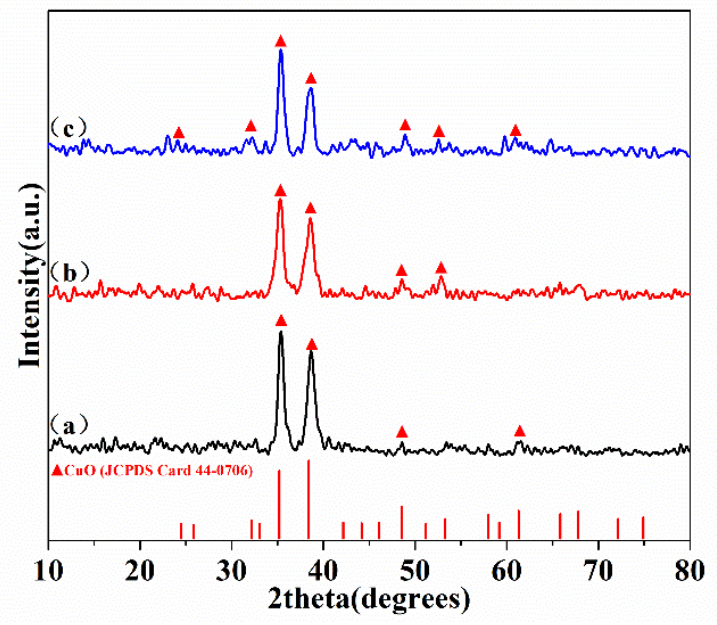

Figure S1. XRD patterns of $\mathrm{CuO} @ \mathrm{NiO}$ nanowires film at different chemical bath deposition times, (a) $5 \mathrm{~min}$; (b) $10 \mathrm{~min}$; (c) $15 \mathrm{~min}$.

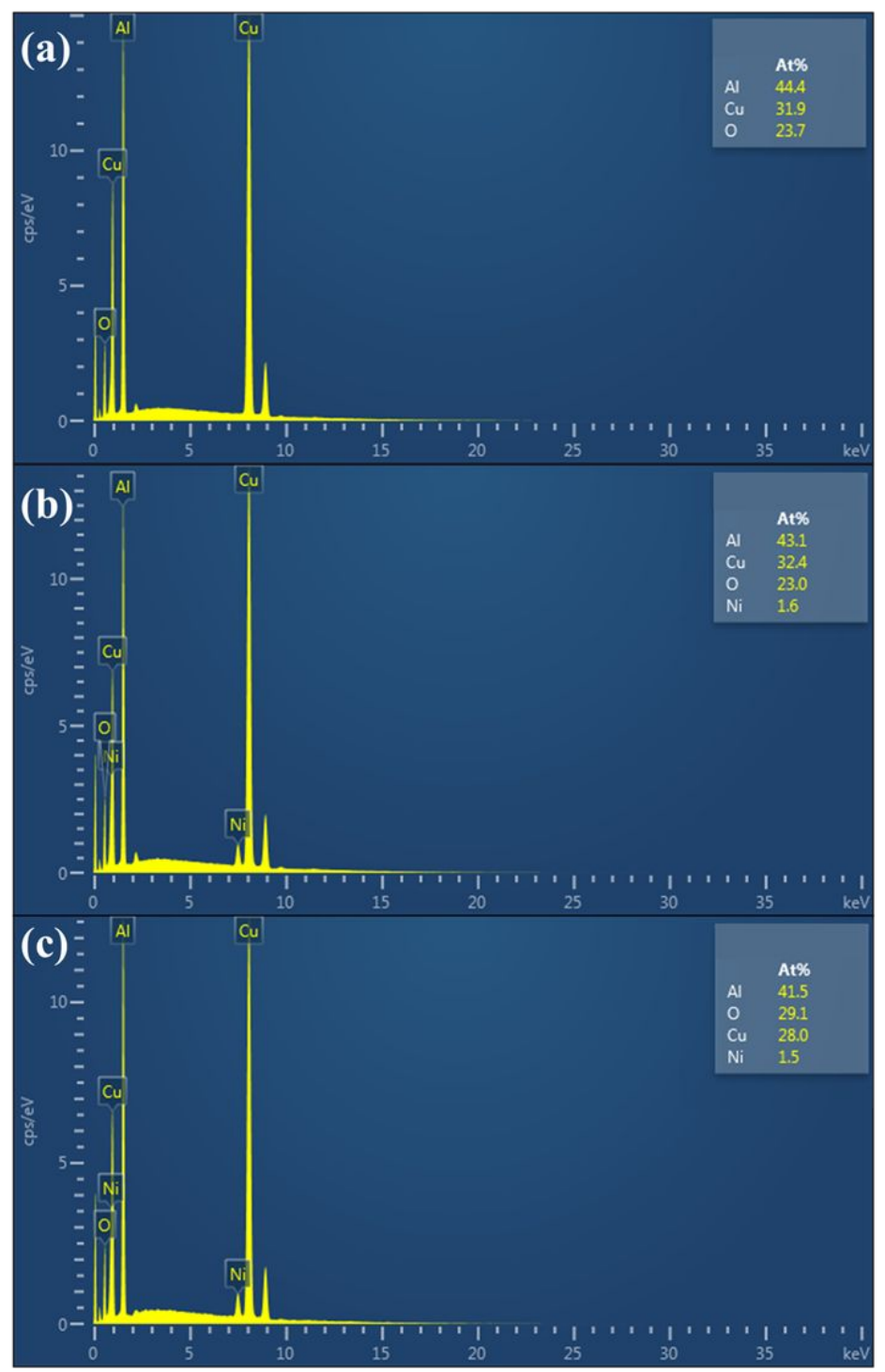

Figure S2. The EDS spectra for the three nanowires thermite film systems of (a) 
CuO/Al, (b) CuO@Ni/Al and (c) CuO@NiO/Al.
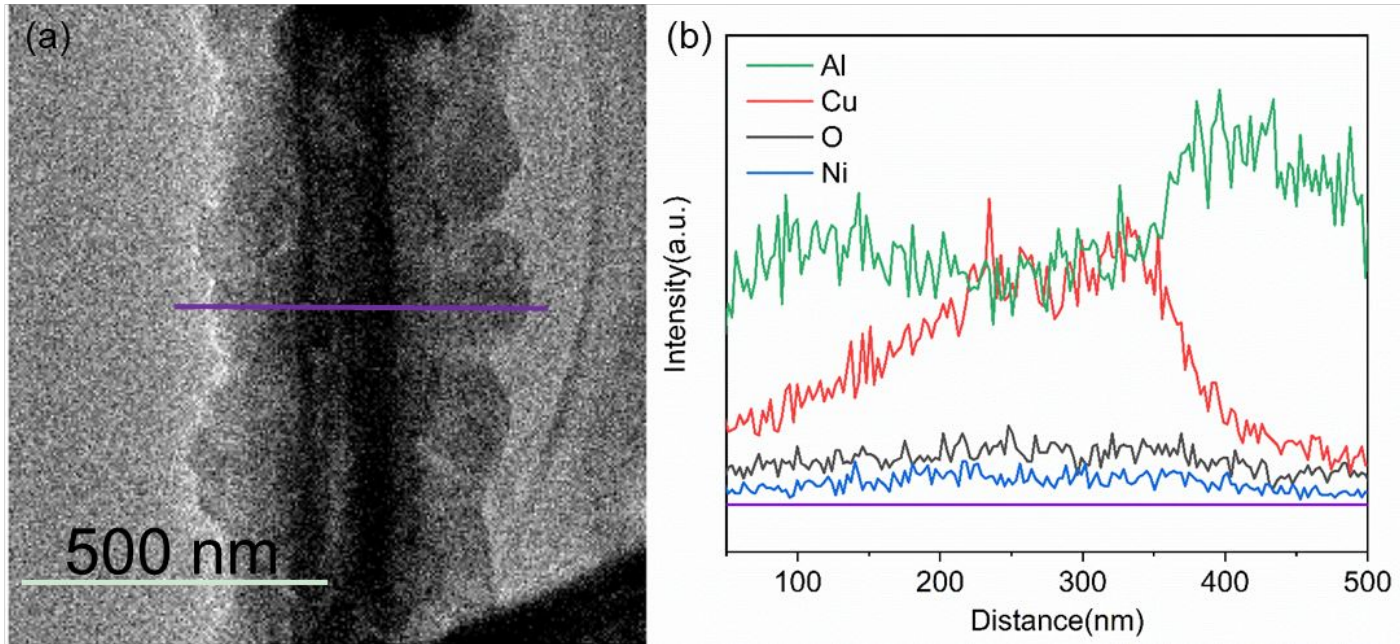

Figure S3. CuO@NiO/Al thermite film systems (a) TEM image, (b) EDS line scan spectra.
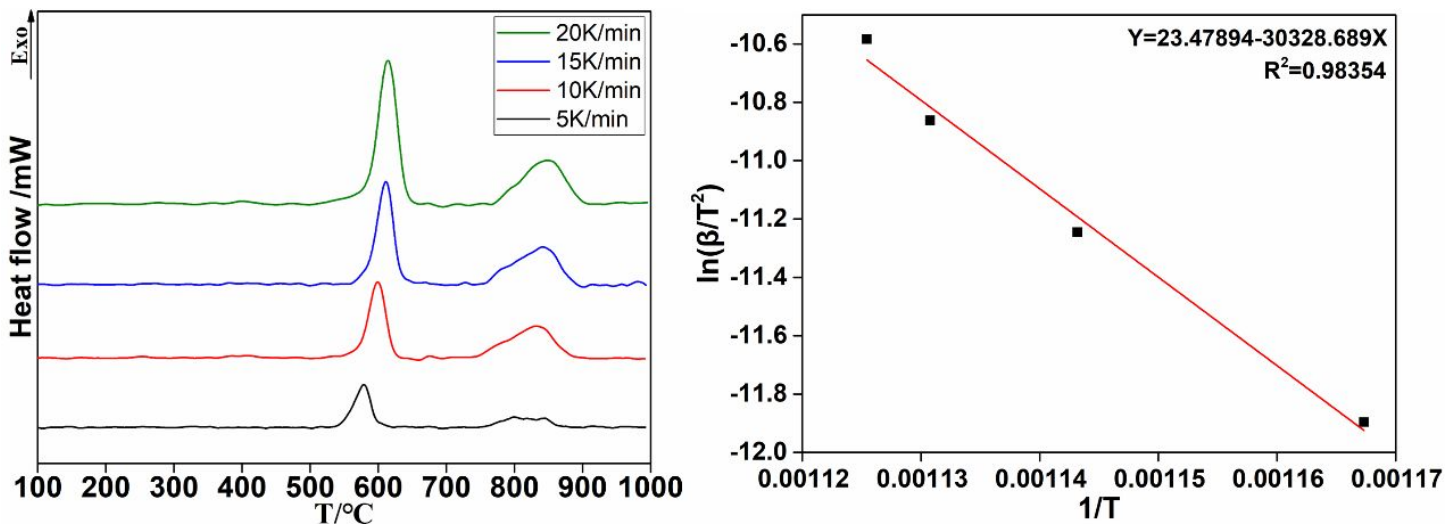

Figure S4. DSC curves of $\mathrm{CuO} / \mathrm{Al}$ at various heating rates and corresponding linear fit result.
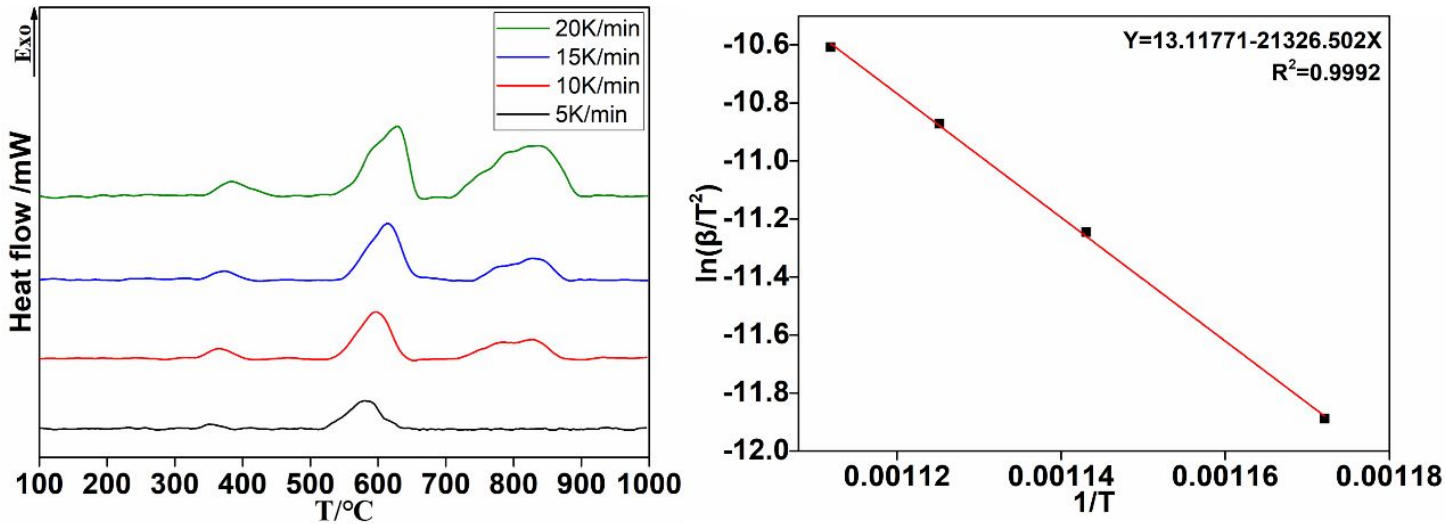

Figure S5. DSC curves of $\mathrm{CuO} @ \mathrm{Ni} / \mathrm{Al}$ at various heating rates and corresponding linear fit result. 

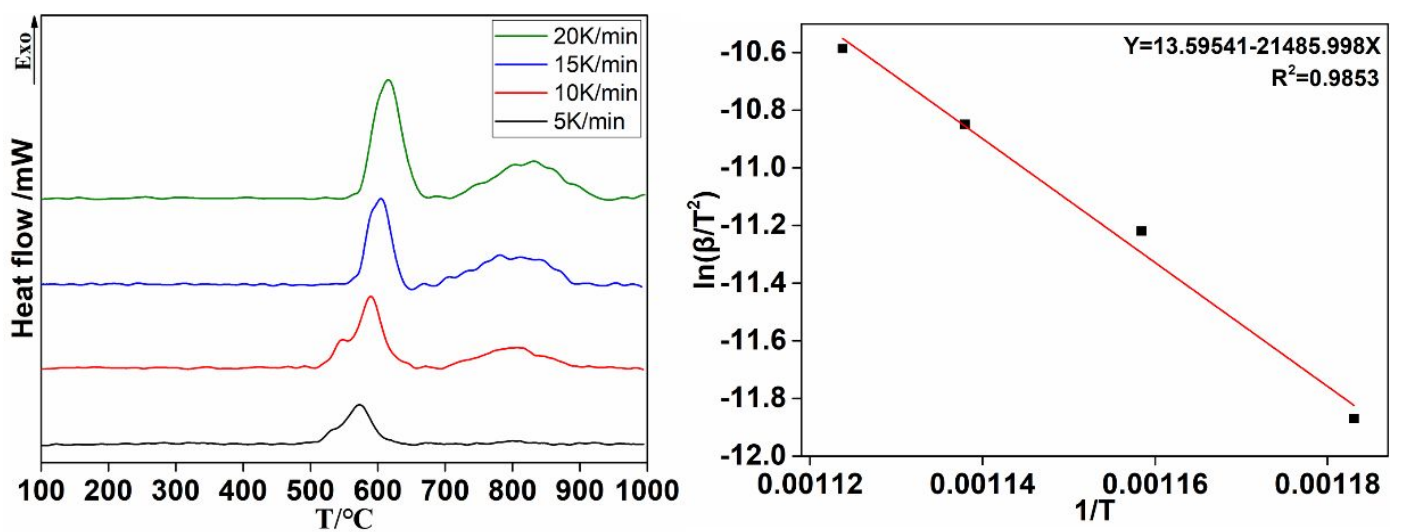

Figure S6. DSC curves of $\mathrm{CuO} @ \mathrm{NiO} / \mathrm{Al}$ at various heating rates and corresponding linear fit result.

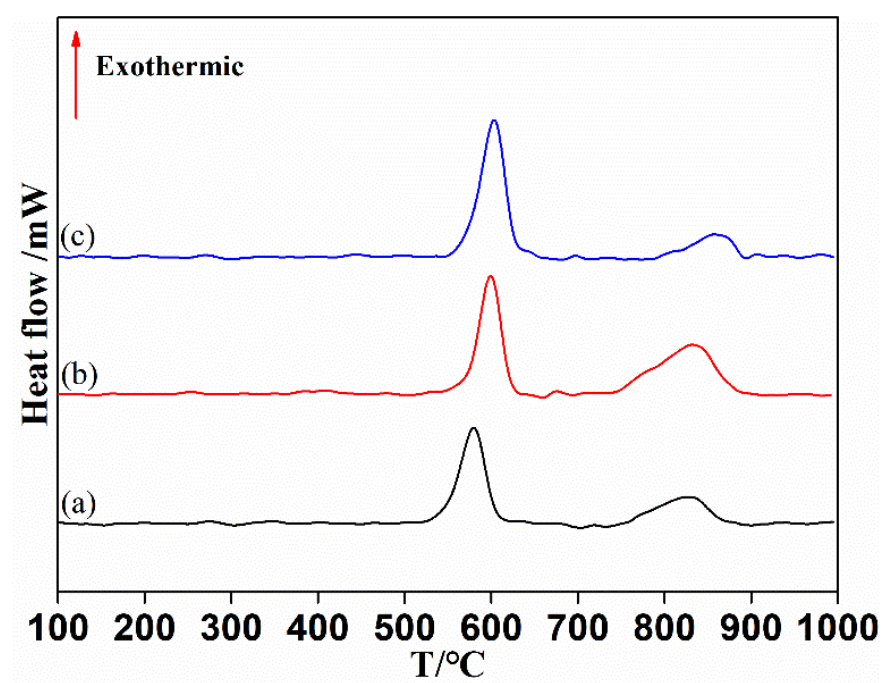

Figure S7. DSC curves of $\mathrm{CuO} / \mathrm{Al}$ at different $\mathrm{Al}$ deposition time, (a) $15 \mathrm{~min}$; (b) 30 min; (c) 45 min. 